\title{
Option Pricing for GARCH-type Models with Generalized Hyperbolic Innovations*
}

\author{
${\text { Christophe } \text { Chorro }^{\dagger} \quad \text { Dominique Guégan }}^{\ddagger} \quad$ Florian Ielpo $^{\S}$
}

May 11, 2010

\begin{abstract}
In this paper, we provide a new dynamic asset pricing model for plain vanilla options and we discuss its ability to produce minimum mispricing errors on equity option books. Given the historical measure, the dynamics of assets are modeled by Garch-type models with generalized hyperbolic innovations and the pricing kernel is an exponential affine function of the state variables, we show that the risk neutral distribution is unique and implies again a generalized hyperbolic dynamics with changed parameters. We provide an empirical test for our pricing methodology on two data sets of options respectively written on the French CAC 40 and the American SP 500. Then, using our theoretical result associated with Monte Carlo simulations, we compare this approach to natural competitors in order to test its efficiency. More generally, our empirical investigations analyze the ability of specific parametric innovations to reproduce market prices in the context of an exponential affine specification of the stochastic discount factor.
\end{abstract}

Keywords: Generalized hyperbolic distribution, Option pricing, Incomplete markets, CAC 40, SP 500, GARCH-type models.

JEL classification: G13, C22.

\footnotetext{
${ }^{*}$ The authors are thankful to seminar participants of the Economics departments at the Universities of Bergamo (Italy) and Aarhus (Denmark), of the Mathematics departments of QUT in Brisbane (Australia), UNSW in Sydney (Australia), CSIRO in Melbourne (Australia) and Hong Kong University and finally to the local Paris 1 Sorbonne seminar for their questions and remarks. This paper has also been presented at the International Symposium on Forecasting in Nice (France) and as a keynote speech at the third International Forum on Intelligence Finance in Beijing (China). We also thank two anonymous referees for their comments and remarks that helped us to improve the quality of this article. The usual disclaimer nonetheless applies.

${ }^{\dagger}$ Université Paris 1, Centre d'Economie de la Sorbonne, Maison des Sciences Economiques, 106 bd de l'Hôpital, 75013 Paris, France. Email: cchorro@univ-paris1.fr.

${ }^{\ddagger}$ Corresponding author.Université Paris 1, Centre d’Economie de la Sorbonne, Maison des Sciences Economiques, 106 bd de l'Hôpital, 75013 Paris, France. Email: dguegan@univ-paris1.fr.. Tel: +33 140 078298.

${ }^{\S}$ Université Paris 1, Centre d'Economie de la Sorbonne, Maison des Sciences Economiques, 106 bd de l'Hôpital, 75013 Paris, France. Email: florian.ielpo@ensae.org.
} 


\section{Introduction}

In this paper, we provide a new dynamic asset pricing model for plain vanilla options and we discuss its ability to produce minimum mispricing errors on equity option books. Given the historical measure, the dynamics of assets are modeled by Garch-type models with generalized hyperbolic innovations and the pricing kernel is an exponential affine function of the state variables, we show that the risk neutral distribution is unique and implies again a generalized hyperbolic dynamics with changed parameters. We provide an empirical test for our pricing methodology on two data sets of options respectively written on the French CAC 40 and the American SP 500. Then, using our theoretical result associated with Monte Carlo simulations, we compare this approach to natural competitors in order to test its efficiency. More generally, our empirical investigations analyze the ability of specific parametric innovations to reproduce market prices in the context of an exponential affine specification of the stochastic discount factor.

Since the celebrated Black-Scholes formula for pricing call options under constant volatility, researchers have paid attention to more general models to explain some well-known mispricing phenomena. It is now generally admitted that returns exhibit time-varying conditional variance, leading to the building of models consistent with this stylized fact, either in a continuous or a discrete time setting. Examples include Heston (1993), Engle (1982) or Bollerslev (1986). Unfortunately, incompleteness can arise through these extensions of the Black-Scholes economy which is problematic. Therefore, in an incomplete market with no arbitrage opportunities, there is more than one equivalent martingale measure and hence a range of no-arbitrage prices for a contingent claim. Thus, one crucial issue is to identify an equivalent martingale measure which gives economically consistent prices for contingent claims.

By using an equilibrium argument, Duan (1995) gave an economically consistent approach to options pricing in related GARCH models with normal innovations. Following this methodology, Heston and Nandi (2000) considered a new conditionally-normal GARCH model able to cope with skews in option prices. They derived an almost closed form expression for call option prices and empirically demonstrated its pricing performance. As the model is conditionally normal, it usually fails to capture the short term behavior of equity option smiles. In fact, the importance of asymmetry and heavy tails inside financial time series is now well documented in the literature, as in Bouchaud and Potters (2003) or Embrechts et al. (2005) especially for pricing issues.

To settle the latter problem, we proceed as follows:

First, under the historical distribution $\mathbb{P}$, we use GARCH-type discrete time modeling for the underlying asset, with an appropriate distribution (here the class of generalized hyperbolic distributions) for the innovations to take into account the most important features which characterize financial time series, such as skewness and kurtosis. The generalized hyperbolic 
distribution introduced by Barndorff-Nielsen (1977) is known to fit financial data sets remarkably. It can handle especially the particular tail behavior found in equity index returns (see Section 2). It has already been used with empirical success to model the dynamics of several stock markets in discrete or continuous time (for example, in Eberlein and Prause (2002) or Guégan and Zhang (2009)).

Second, we have to select a particular risk neutral probability measure with an interesting economic interpretation. One of the major result in this area was proposed by Gerber and Shiu (1994b), who provide an elegant way to choose an equivalent martingale measure using the Esscher transform. This tool was introduced in actuarial science by Esscher (1932) in an incomplete market setting. In contrast to Duan's (1995) approach, this latter framework permits a wide variety of return innovations to be chosen within the class of infinite, divisible distributions. This method has already been used to price options in discrete financial models as in Bühlmann et al. (1996), Siu et al. (2004) or Christoffersen et al. (2006), and even in continuous time for Lévy-type ones as in Eberlein and Prause (2002). An equivalent formulation of the work of Gerber and Shiu (1994b) consists in using an exponential affine parametrization for the classical stochastic discount factor. In this article, we show that in the framework of GARCH-type models with generalized hyperbolic innovations, this particular change of probability implies again a generalized hyperbolic dynamics with explicit parameters allowing for Monte Carlo simulations.

Lastly, we compare the out-of-sample pricing performances of the GARCH-type models with generalized hyperbolic innovations by competitors. On the one hand, the Black and Scholes (1973) and Heston and Nandi (2000) models built on the normal distribution appear as interesting benchmarks because, for each of them, the exponential affine form of the pricing kernel is assumed (implicitly or explicitly) to move from the historical distribution to the risk neutral one. On the other hand, we also consider GARCH-type models with mixtures of normal distributions combined with an Esscher transform. The use of a mixture of two normal distributions to model the innovations may be interestingly compared to our choice of the generalized hyperbolic distribution, because these two families have exactly the same number of parameters. Moreover this kind of model has been recently introduced by Alexander and Lazar (2006), offering a better fit than the classical GARCH process with a normal distribution. With this study, we want to highlight the interest of the choice of the generalized hyperbolic distribution to price options, especially when it is associated with GARCH models.

The paper is organized as follows. In Section 2, we describe the data sets on which we test our model. We state the classical properties of the generalized hyperbolic distribution and present the models we use under the historical distribution. Section 3 is devoted to the choice of the stochastic discount factor and thus of the risk neutral measure. In Section 4 , we discuss the volatility structures to choose for empirics, we describe the option pricing methodology based on Monte Carlo simulations and we analyze the empirical results obtained for the French CAC 40 and the American SP 500 indexes, to compare this approach with 
other classical models. Section 5 concludes and proposes some possible extensions. All the proofs are given in the appendix.

\section{The Generalized Hyperbolic Distribution for Modeling Fi- nancial Returns}

In this Section, we discuss the interest of modeling financial returns using the generalized hyperbolic distribution. First, we present the data sets used in the article. Then, we provide a short introduction to the generalized hyperbolic distribution. Finally, we present time series models for index returns under the historical distribution.

\subsection{Presentation of the data sets}

The data sets that we use contain the following time series. We consider daily log-returns of the French CAC 40 and the American SP 500 indexes whose values at time $t$ are equally denoted $S_{t}$. We use closing prices for both indexes. The samples start on January 2, 1988 and end on October 26, 2007. Elementary descriptive statistics are provided in Table 1.

[Table 1 about here]

Following this preliminary description we test various probability distributions in order to find an interesting version. In this paper we are particularly interested in the generalized hyperbolic distribution developed by Barndorff-Nielsen (1977). The next Subsection presents its definition in a formal way. This type of distribution has already been suggested as a model for financial price processes as its exponentially decreasing tails seem to fit the statistical behavior of asset returns remarkably, as noted in Barndorff-Nielsen (1995) or Eberlein and Prause (2002). The results are presented in Table 2.

[Table 2 about here]

From the results provided in Table 2 we observe that the normal distribution is strongly rejected as a model for the conditional distributions of the CAC 40 and the SP 500 log-returns. In contrast, the distributions belonging to the generalized hyperbolic family give rise to satisfactory results. We provide several figures that confirm these results. In Figure 1, we present the empirical log-density (plain black line) vs. the estimated log-density obtained with the NIG (red), Hyperbolic (green), Generalized Hyperbolic (dark blue) and Normal (light blue) distributions for the daily log-returns of each index. In Figure 2, we present the qq-plots comparing the empirical quantiles of indexes returns vs. the estimated quantiles obtained with the NIG (red), Hyperbolic (green), Generalized Hyperbolic (dark blue) and Normal (light blue) distributions. Based on the graphics and the tables previously mentioned, it is natural that a dynamic asset pricing model is based on a distribution that belongs to the generalized hyperbolic family. 
[Figure 1 about here]

[Figure 2 about here]

Financial theory states that the price of any asset is equal to the present value of the expected payoff, under a well chosen distribution. Thus, to compute option prices, we need to know the risk-free rate that can be used to compute the necessary discount factors. The risk free rate used in this paper is the zero coupon rate obtained from the European and US interest rates swap (OIS). We use closing swap rates whose maturities range from 1 month to 3 years. Intermediate maturities required for option pricing are computed using the Svensson (1994) model. ${ }^{1}$

Beyond these initial data sets, we use the available option contracts written on the indexes. For the CAC 40 and the SP 500, the most liquid contracts available are the quarterly ones, i.e. the contracts maturing in March, June, September and December, for all available years. We focused on these maturities, neglecting the intermediate monthly maturities that are far less liquid on average. The option data sets start on January 2, 2006 and end on October 26, 2007. The strike prices are chosen so that the moneynesses used here ranges from 0.8 to 1.2, which is the standard moneyness window used in the literature. Table 3 presents key statistics regarding the option data sets across moneynesses and maturities ${ }^{2}$.

[Table 3 about here]

\subsection{The generalized hyperbolic distribution}

In this Subsection, we specify properties of the generalized hyperbolic distribution which are useful in the following.

The Generalized Hyperbolic (GH) distribution is particularly interesting characterizing the exponentially decreasing tails which are often observed in asset returns.

For $(\lambda, \alpha, \beta, \delta, \mu) \in \mathbb{R}^{5}$ with $\delta>0$ and $\alpha>|\beta|>0$, the one dimensional $G H(\lambda, \alpha, \beta, \delta, \mu)$ distribution is defined by the following density function:

$$
d_{G H}(x, \lambda, \alpha, \beta, \delta, \mu)=\frac{\left(\sqrt{\alpha^{2}-\beta^{2}} / \delta\right)^{\lambda}}{\sqrt{2 \pi} K_{\lambda}\left(\delta \sqrt{\alpha^{2}-\beta^{2}}\right)} e^{\beta(x-\mu)} \frac{K_{\lambda-1 / 2}\left(\alpha \sqrt{\delta^{2}+(x-\mu)^{2}}\right)}{\left(\sqrt{\delta^{2}+(x-\mu)^{2}} / \alpha\right)^{1 / 2-\lambda}}
$$

where $K_{\lambda}$ is the modified Bessel function of the third kind. For $\lambda \in \frac{1}{2} \mathbb{Z}$, the basic properties of the Bessel function allow simpler forms for the density to be found. In particular, for $\lambda=1$,

\footnotetext{
${ }^{1}$ We compared the results obtained with the Nelson and Siegel (1987) approach and the performances of the models presented here are generally unchanged.

${ }^{2}$ Finally, when implementing the pricing formula proposed in Section 3 the dividends paid by the indexes have to be taken into account in the case of the SP 500. In this paper we use the dividends classically extracted from the option data sets considering the call-put parity.
} 
we get the Hyperbolic distribution (HYP) whose log-density is a hyperbola. For $\lambda=-\frac{1}{2}$, we obtain the Normal Inverse Gaussian distribution (NIG) which is closed under convolution. The moment generating function of a $G H$ distribution exists and is given by:

$$
\mathbb{G}_{G H}(u)=e^{\mu u}\left(\frac{\alpha^{2}-\beta^{2}}{\alpha^{2}-(\beta+u)^{2}}\right)^{\frac{\lambda}{2}} \frac{K_{\lambda}\left(\delta \sqrt{\alpha^{2}-(\beta+u)^{2}}\right)}{K_{\lambda}\left(\delta \sqrt{\alpha^{2}-\beta^{2}}\right)}, \quad|\beta+u|<\alpha .
$$

In particular, moments of all orders are finite allowing Central Limit Theorem arguments to be applied to ensure the convergence of long time horizon returns on Normal distributions. Finally, this family is also stable under affine transforms, as proved in Blaesild (1981). This property is interesting because in the GARCH setting we will be able to deduce the conditional distribution of the log-returns from the innovations. In particular, we define $\alpha^{*}=\alpha \delta$ and $\beta^{*}=\beta \delta$ and if $X \hookrightarrow G H\left(\lambda, \alpha^{*}, \beta^{*}, \delta, \mu\right)$ then $\frac{X-\mu}{\delta} \hookrightarrow G H(\lambda, \alpha, \beta, 1,0)$ : the parameters $\mu$ and $\delta$ respectively describe the location and the scale.

\subsection{Description of the economy under the historical probability $\mathbb{P}$}

Well-documented empirical evidence suggests that equity return volatility is stochastic and mean reverting. In the discrete time setting, the stochastic volatility is often captured with extensions of the autoregressive conditional heteroscedasticity model (ARCH) that will be used in this paper, like the Nelson (1991)'s EGARCH model or the APARCH model of Ding et al. (1993).

Let $\left(z_{t}\right)_{t \in\{0,1, \ldots, T\}}$ be independent identically distributed random variables defined on the sample space $(\Omega, \mathcal{A}, \mathbb{P})$ and $\left(\mathcal{F}_{t}=\sigma\left(z_{u} ; 0 \leq u \leq t\right)\right)_{t \in\{0,1, \ldots, T\}}$ the associated information filtration.

We assume that under the historical probability $\mathbb{P}$, the dynamics of the bond price process $\left(B_{t}\right)_{t \in\{0,1, \ldots, T\}}$ and the stock price process $\left(S_{t}\right)_{t \in\{0,1, \ldots, T\}}$ are given by

$$
B_{t}=B_{t-1} e^{r}, \quad B_{0}=1,
$$

where $r$ is the corresponding risk free rate expressed on a daily basis, supposed to be constant and:

$$
Y_{t}=\log \left(\frac{S_{t}}{S_{t-1}}\right)=r+m_{t}+\underbrace{\sqrt{h_{t}} z_{t}}_{\varepsilon_{t}}, \quad S_{0}=s,
$$

where $z_{t} \hookrightarrow f(0,1)$ ( $f$ being an arbitrary distribution with mean 0 and variance 1 , which will be the generalized hyperbolic distribution found empirically). In equation (4), we consider a general time varying excess of return $m_{t}$ that depends on the constant unit risk premium $\lambda_{0}$. In practice, it will be fixed and in this paper we used the following representation:

$$
m_{t}=\lambda_{0} \sqrt{h_{t}}-\frac{1}{2} h_{t}
$$

For the conditional variance of the returns we choose a GARCH-type model of the form

$$
h_{t}=F\left(z_{t-1}, h_{t-1}\right)
$$


that is not specified for the moment. In Section 4, we discuss the choice of $h_{t}$ in order to take into account the most important features of the data sets we consider: in particular to capture asymmetry phenomena and to be consistent with the well-known leverage effects observed in financial markets.

According to Blaesild (1981), the conditional distribution of $Y_{t}$ may now be deduced from the $z_{t}$ one, given $\mathcal{F}_{t-1}$,

$$
Y_{t} \hookrightarrow G H\left(\lambda, \frac{\alpha}{\sqrt{h_{t}}}, \frac{\beta}{\sqrt{h_{t}}}, \delta \sqrt{h_{t}}, r+m_{t}+\mu \sqrt{h_{t}}\right) .
$$

Thus, for the estimation of the GARCH-type model under the historical probability, we may adopt a two-stage procedure. In the first stage, the Quasi Maximum Likelihood Estimation is used to determine the parameters of the volatility function $F$. In the second stage, since we exactly know the form of the density function (1) we adopt a classical maximum likelihood approach to estimate the unknown remaining parameters $(\lambda, \alpha, \beta, \delta, \mu)$.

Now, our model is entirely specified under $\mathbb{P}$. Since we want to use it to price contingent claims, we need to postulate an explicit risk premium to perform the change in distribution. This is the aim of the next part.

\section{Pricing Methodology}

This Section is devoted to the option pricing methodology. We first present the stochastic discount factor option pricing framework. Then, we discuss the hypothesis of an exponential affine stochastic discount factor for option pricing. Finally, we apply this framework to the generalized hyperbolic distribution. The proofs of the results are given in an appendix at the end of the paper.

\subsection{The choice of the stochastic discount factor}

We consider the preceding economy with time horizon $T$ consisting of two assets, namely a risk-free bond and a risky stock. It should be recalled that we denote the dynamics of the bond by $\left(B_{t}\right)_{t \leq T}$, and the stock price processes by $\left(S_{t}\right)_{t \leq T}$, under the historical probability $\mathbb{P}$.

Classically, in a discrete time dynamic equilibrium model (or in a continuous model free of arbitrage), the price of any asset equals the expected present value of its future payoffs under an equivalent martingale measure $\mathbb{Q}$. For example, the price $P_{t}$ at time $t$ of a European asset paying $\Phi_{T}$ at $T$ ( $\Phi_{T}$ being $\mathcal{F}_{T}$ measurable) is given by

$$
P_{t}=E_{\mathbb{Q}}\left[\Phi_{T} e^{-r(T-t)} \mid \mathcal{F}_{t}\right]
$$

or equivalently

$$
P_{t}=E_{\mathbb{P}}\left[\Phi_{T} M_{t, T} \mid \mathcal{F}_{t}\right]
$$


The $\mathcal{F}_{t+1}$ measurable random variable $M_{t, t+1}$ is the so-called stochastic discount factor (the quantity $M_{t, t+1} e^{r}$ is also known as the pricing kernel). In general, the stochastic discount factor depends on several structural variables of the economy, such as past consumption or equity market returns. Nevertheless, following Rubinstein (1976) or Cochrane (2001) we suppose that equity market returns are the only variables to deal with pricing purposes or equivalently that we may project the original stochastic discount factor onto the sigma-algebra, generated by the payoffs of the risky asset.

In discrete time, it is well known that markets are in general incomplete. Thus, the martingale measure $\mathbb{Q}$ is not unique and there exists a multiplicity of stochastic discount factors that are compatible with the previous pricing formulas.

Once the dynamics under the historical probability have been specified throughout statistical modelings, we may overcome this problem adopting one of the two following equivalent points of view: we may impose some constraints on the form of the stochastic discount factor or choose a particular martingale measure that fulfills some economic or risk criteria (e.g the minimal martingale measure in the sense of Fôllmer and Schweiser (1991) that minimizes the variance of the hedging loss). When this choice has been made, if we know the dynamics of the risky asset under the new probability, then it is possible to price contingent claims from (8) or (9) using Monte Carlo simulations.

To solve these two points, we follow the work of Gerber and Shiu (1994b) choosing for the stochastic discount factor an exponential affine parametrisation and we prove that starting from a GARCH-type model with generalized hyperbolic innovations under $\mathbb{P}$, we obtain again under $\mathbb{Q}$ a generalized hyperbolic dynamics with explicit parameters allowing for Monte Carlo simulations. We now develop our method.

\subsection{Pricing options with exponential affine stochastic discount factors}

The methodology unfolds as follows. We assume for the stochastic discount factor a particular parametric form: $\forall t \in\{0, \ldots, T-1\}$

$$
M_{t, t+1}=e^{\theta_{t+1} Y_{t+1}+\xi_{t+1}}
$$

where $Y_{t+1}=\log \left(\frac{S_{t+1}}{S_{t}}\right)$ and where $\theta_{t+1}$ and $\xi_{t+1}$ are $\mathcal{F}_{t}$ measurable random variables.

Recall that in a discrete time version of the Black-Scholes economy, the corresponding stochastic discount factor is given by $\theta_{t+1}=\frac{\mu-r}{\sigma^{2}}$ and $\xi_{t+1}=\frac{\left(\sigma^{2}-r-\mu\right)(\mu-r)}{2 \sigma^{2}}$ that are independent of $t$. In particular, the parameter $\theta$ corresponds to a constant risk aversion. Here, the specification (10) allows for time variation in risk aversion.

We need to compute explicitly $\left(\theta_{t+1}, \xi_{t+1}\right)$. Considering the bond and the risky asset, the pricing relation (9) for $T=t+1$ gives the following restrictions for the stochastic discount 
factor

$$
\left\{\begin{array}{l}
E_{\mathbb{P}}\left[e^{r} M_{t, t+1} \mid \mathcal{F}_{t}\right]=1 \\
E_{\mathbb{P}}\left[e^{Y_{t+1}} M_{t, t+1} \mid \mathcal{F}_{t}\right]=1 .
\end{array}\right.
$$

For all $t \in\{0, \ldots, T-1\}$, we denote by $\mathbb{G}_{t}$ the conditional moment generating function of $Y_{t+1}$, given $\mathcal{F}_{t}$ defined on a convex set $\mathcal{D}_{\mathbb{G}_{t}}$ which is not reduced to $\{0\}$, and by $\Theta_{t}$ the parameter set $\left\{\theta \in \mathbb{R} ; \theta\right.$ and $\left.1+\theta \in \mathcal{D}_{\mathbb{G}_{t}}\right\}$. We now introduce the mapping $\Phi_{t}: \Theta_{t} \rightarrow \mathbb{R}$ such that

$$
\Phi_{t}(\theta)=\log \left(\frac{\mathbb{G}_{t}(1+\theta)}{\mathbb{G}_{t}(\theta)}\right) .
$$

Thus, the preceding system is equivalent to

$$
\left\{\begin{array}{l}
\mathbb{G}_{t}\left(\theta_{t+1}\right)=e^{-\left(r+\xi_{t+1}\right)} \\
\mathbb{G}_{t}\left(\theta_{t+1}+1\right)=e^{-\xi_{t+1}}
\end{array}\right.
$$

and, with our notations, we have to solve

$$
\left\{\begin{array}{l}
\Phi_{t}\left(\theta_{t+1}\right)=r \\
\mathbb{G}_{t}\left(\theta_{t+1}+1\right)=e^{-\xi_{t+1}} .
\end{array}\right.
$$

The next proposition shows that, under the pricing constraints (11), there is no ambiguity in the choice of the stochastic discount factor (10).

Proposition 1. Suppose that $\mathbb{G}_{t}$ is twice differentiable. If there exists a solution to the equation $\Phi_{t}(\theta)=r$, it is unique.

Proof: See Gerber and Shiu (1994a)

Subsequently, we suppose for each $t \in\{0, \ldots, T-1\}$ that relationship (13) leads to a unique solution denoted by $\left(\theta_{t+1}^{q}, \xi_{t+1}^{q}\right)$ (in the next Subsection we provide the proof of existence for the GH distribution). The stochastic discount factor

$$
M_{t, t+1}=e^{\theta_{t+1}^{q} Y_{t+1}+\xi_{t+1}^{q}}
$$

being known explicitly, we may deduce easily the form of the associated equivalent martingale measure $\mathbb{Q}$. In fact, remarking that $\forall k \in\{0, \ldots, T-1\}$

$$
\frac{M_{k, k+1}}{E_{\mathbb{P}}\left[M_{k, k+1} \mid \mathcal{F}_{k}\right]}=\frac{e^{\theta_{k+1}^{q} Y_{k+1}}}{\mathbb{G}_{k}\left(\theta_{k+1}^{q}\right)},
$$

we define the stochastic process

$$
\left(L_{t}=\prod_{k=1}^{t} \frac{e^{\theta_{k}^{q} Y_{k}}}{\mathbb{G}_{k-1}\left(\theta_{k}^{q}\right)}\right)_{t \in\{1, \ldots, T\}}
$$

that is obviously a martingale under $\mathbb{P}$. Then, we obtain the following proposition: 
Proposition 2. Let $\mathbb{Q}$ be the probability with a density $L_{T}$ with respect to $\mathbb{P}$. Then,

a) $\mathbb{Q}$ is the unique probability associated with the exponential affine stochastic discount factor (14), in particular, the discounted stock price process $\left(e^{-r t} S_{t}\right)_{t \in\{0, \ldots, T\}}$ is a martingale under $\mathbb{Q}$ and the price $P_{t}$ at time $t$ of a European asset paying $\Phi_{T}$ at $T$ is given by

$$
P_{t}=E_{\mathbb{Q}}\left[\Phi_{T} e^{-r(T-t)} \mid \mathcal{F}_{t}\right] .
$$

b) Under $\mathbb{Q}$, the moment generating function of $Y_{t}$ given $\mathcal{F}_{t-1}$ is given by

$$
E_{\mathbb{Q}}\left[e^{u Y_{t}} \mid \mathcal{F}_{t-1}\right]=E_{\mathbb{P}}\left[e^{u Y_{t}} \frac{e^{\theta_{t}^{q} Y_{t}}}{\mathbb{G}_{t-1}\left(\theta_{t}^{q}\right)} \mid \mathcal{F}_{t-1}\right]=\frac{\mathbb{G}_{t-1}\left(\theta_{t}^{q}+u\right)}{\mathbb{G}_{t-1}\left(\theta_{t}^{q}\right)} .
$$

Under $\mathbb{Q}$, the conditional distribution of $Y_{t}$ given $\mathcal{F}_{t-1}$ is none other than the conditional Esscher transform of parameter $\theta_{t}^{q}$ of the distribution of $Y_{t}$ given $\mathcal{F}_{t-1}$ under $\mathbb{P}$. Moreover, for pricing purposes, relation (17) is fundamental because it gives explicitly the conditional distribution of the log-returns under $\mathbb{Q}$ and allows for Monte Carlo simulation methods. Furthermore, as underlined in the next proposition, under weak assumptions, this conditional distribution under $\mathbb{Q}$ belongs to the same family as under the historical one.

Proposition 3. For all $t \in\{1, \ldots, T\}$, if the conditional distribution of $Y_{t}$ given $\mathcal{F}_{t-1}$ is infinitely divisible under $\mathbb{P}$ and if $\mathbb{G}_{t-1}$ is twice differentiable, then, the conditional distribution of $Y_{t}$ given $\mathcal{F}_{t-1}$ is also infinitely divisible under $\mathbb{Q}$ with a finite moment of order 2.

The preceding result is not so surprising because several authors have already remarked that it is true for particular distributions as in Siu et al. (2004) or Christoffersen et al. (2006). This point is one of the main features of the exponential affine specification of the pricing kernel that is not fulfilled, for example, in the framework of Elliot and Madan (1998). For the class of GH distributions the stability is proved in the next Subsection.

\subsection{Option pricing for GARCH-type models with GH innovations}

We apply the methodology of the preceding Subsection using the GH setting. Thus, we have to identify (if it exists) the unique exponential affine stochastic discount factor and describe explicitly the dynamics of the log-returns under the associated equivalent martingale measure.

First, we obtain a result that ensures, under weak conditions, the existence of a solution $\left(\theta_{t+1}^{q}, \xi_{t+1}^{q}\right)$ of $(13)$ for all $t \in\{0, \ldots, T-1\}$.

Proposition 4. For a $G H(\lambda, \alpha, \beta, \delta, \mu)$ distribution with $\alpha>\frac{1}{2}$, then,

a) If $\lambda \geq 0$, the equation $\log \left(\frac{\mathbb{G}_{G H}(1+\theta)}{\mathbb{G}_{G H}(\theta)}\right)=r$ has a unique solution,

b) If $\lambda<0$, the equation $\log \left(\frac{\mathbb{G}_{G H}(1+\theta)}{\mathbb{G}_{G H}(\theta)}\right)=r$ has a unique solution if and only if $\mu-C<$ $r<\mu+C$ where

$$
C=\log \left(\frac{\Gamma[-\lambda]}{2^{\lambda+1}}\right)-\log \left(\frac{K_{\lambda}\left(\delta \sqrt{\alpha^{2}-(\alpha-1)^{2}}\right)}{\delta \sqrt{\alpha^{2}-(\alpha-1)^{2}}}\right)
$$


The constant $C$ is strictly positive because $\frac{d}{d x} \frac{K_{\lambda}(x)}{x^{\lambda}}=-\frac{K_{\lambda+1}(x)}{x^{\lambda}}<0$.

Even if we are not able to obtain a closed form formula for the solution of (13), we may apply the risk neutral valuation presented in Section 3.2. For practical purposes, $\left(\theta_{t+1}^{q}, \xi_{t+1}^{q}\right)$ may be computed efficiently using a refined bracketing method.

The next proposition describes the dynamics of the risky asset under the chosen equivalent martingale measure. It derives from the Proposition 2.

Proposition 5. Under $\mathbb{Q}$, the distribution of $Y_{t}$ given $\mathcal{F}_{t-1}$ is a

$$
G H\left(\lambda, \frac{\alpha}{\sqrt{h_{t}}}, \frac{\beta}{\sqrt{h_{t}}}+\theta_{t}^{q}, \delta \sqrt{h_{t}}, r+m_{t}+\mu \sqrt{h_{t}}\right) .
$$

It is interesting to notice that the parameter $\xi_{t}^{q}$ in the stochastic discount factor does not appear in the stock price dynamics under the equivalent martingale measure $\mathbb{Q}$. Moreover, the appearance of $\theta_{t}^{q}$ induces not only a shift in the skewness of the GH distribution but also an excess kurtosis (exact values of the skewness and kurtosis are provided in Barndorff-Nielsen and Blaesild (1981)).

We deduce from the preceding result that, under $\mathbb{Q}$,

$$
Y_{t}=r+m_{t}+\underbrace{\sqrt{h_{t}} z_{t}}_{\varepsilon_{t}}, \quad S_{0}=s,
$$

where the $z_{t}$ are $\mathcal{F}_{t}$ measurable random variables such that, conditionally to $\mathcal{F}_{t-1}$,

$$
z_{t} \hookrightarrow G H\left(\lambda, \alpha, \beta+\sqrt{h_{t}} \theta_{t}^{q}, \delta, \mu\right)
$$

In particular, the $\mathrm{GH}$ distribution is stable given a change of measure, allowing us to simulate easily the sample paths of the risky asset. Under $\mathbb{Q}$, conditionally to $\mathcal{F}_{t-1}, \varepsilon_{t}$ is no longer centered and its variance is not $h_{t}$ but

$$
\operatorname{var}\left(\varepsilon_{t}\right)=h_{t}\left(\frac{\delta K_{\lambda+1}\left(\delta \gamma_{t}\right)}{\gamma_{t} K_{\lambda}\left(\delta \gamma_{t}\right)}+\frac{\left(\beta+\sqrt{h_{t}} \theta_{t}^{q}\right)^{2} \delta^{2}}{\gamma_{t}^{2}}\left(\frac{K_{\lambda+2}\left(\delta \gamma_{t}\right)}{K_{\lambda}\left(\delta \gamma_{t}\right)}-\frac{K_{\lambda+1}^{2}\left(\delta \gamma_{t}\right)}{K_{\lambda}^{2}\left(\delta \gamma_{t}\right)}\right)\right)
$$

where $\gamma_{t}=\sqrt{\alpha^{2}-\left(\beta+\sqrt{h_{t}} \theta_{t}^{q}\right)^{2}}$. Thus the GARCH structure of the volatility is modified in a non-linear way from $\mathbb{P}$ to $\mathbb{Q}$.

\section{Results}

In this Section, we discuss the choice of the conditional volatility structure (6), we detail the empirical methodology for option pricing in our framework and discuss the corresponding results. 


\subsection{The choice of the conditional volatility structure}

We have supposed in Section 2 that the discrete dynamics of the risky asset is given $\forall t \in$ $\{1, \ldots, T\}$ by

$$
Y_{t}=\log \left(\frac{S_{t}}{S_{t-1}}\right)=r+\lambda_{0} \sqrt{h_{t}}-\frac{1}{2} h_{t}+\sqrt{h_{t}} z_{t}, \quad S_{0}=s,
$$

where $z_{t}$ follows a centered generalized hyperbolic distribution with variance 1 and where the conditional variance of the log-returns has the following general GARCH-type form:

$$
h_{t}=F\left(z_{t-1}, h_{t-1}\right) .
$$

In order to improve the pricing performances of our model we propose in this Subsection the results of a test made to select between the best GARCH candidates. To capture asymmetry phenomena observed in our data sets and to be consistent with the well-known leverage effects observed in financial markets we favor three so called candidates:

- the Nelson's (1991) EGARCH model

$$
\log \left(h_{t}\right)=a_{0}+a_{1}\left(\left|z_{t-1}\right|-\gamma z_{t-1}\right)+b_{1} \log \left(h_{t-1}\right)
$$

that ensures positivity without restrictions on the coefficients,

- the Asymmetric Power ARCH model (APARCH) of Ding et al. (1993)

$$
h_{t}^{\Delta}=w+a\left(\left|\sqrt{h_{t-1}} z_{t-1}\right|-\gamma \sqrt{h_{t-1}} z_{t-1}\right)^{\Delta}+b h_{t-1}^{\Delta}
$$

where $\Delta>0$ and $(w, a, b) \in \mathbb{R}_{+}$to ensure positivity,

- the GJR model of Glosten et al. (1993) that is an often used particular case of the APARCH model with $\Delta=2$.

As the models to be compared are not nested, usual likelihood ratio tests are of no use. Rather, we favor the use of the Amisano and Giacomini (2007)'s test, that is based on the test presented in Diebold and Mariano (1995): Say we deal with a time series model for the $\log$-returns whose conditional density at time $t$ is $f_{1}\left(Y_{t} \mid \underline{Y_{t-1}}, \theta_{1}\right)$, where $\underline{Y_{t-1}}=\left(Y_{0}, \ldots, Y_{t-1}\right)$ and $\theta_{1}$ the vector of parameters describing the shape of this conditional distribution and the volatility structure. We compare this model to another one defined by the conditional density $f_{2}\left(Y_{t} \mid Y_{t-1}, \theta_{2}\right)$, with $\theta_{2}$ being the parameters associated to this second model. The null hypothesis of the test is "models 1 and 2 provide a similar fit of the log-return's conditional distribution". The corresponding test statistic used is:

$$
t_{1,2}=\frac{1}{n} \sum_{t=1}^{n}\left(\log f_{1}\left(Y_{t} \mid \underline{Y_{t-1}}, \theta_{1}\right)-\log f_{2}\left(Y_{t} \mid \underline{Y_{t-1}}, \theta_{2}\right)\right)
$$

where $n$ is the total number of observations available. Under the null hypothesis

$$
\frac{t_{1,2}}{\hat{\sigma}_{n}} \sqrt{n} \underset{n \rightarrow+\infty}{\rightarrow} \mathcal{N}(0,1),
$$


where $\hat{\sigma}_{n}$ is a properly selected estimator for the statistic volatility. Here, as proposed in Amisano and Giacomini (2007), we use a Newey-West estimator, with a small lag.

The testing strategy unfolds as follows: first we estimate the three GARCH models on the whole dataset of returns for the CAC 40 and the SP 500 indexes using the two stages estimation strategy explained in details in the next subsection. We focus our attention on a single estimation dealing with the whole sample (from January 2, 1988 to October 26, 2007), as we are interested in understanding the ability of these models to fit the distributional properties of the sample used in the empirical tests performed on option prices. Then, we compute the scaled statistic given in equation (26) for each pair of models. The tables 4 and 5 provide the results for the CAC 40 and the SP 500 indexes. Two key knowledges can be obtained while reading the results: first, there is a shared evidence that the GARCH-GJR model is globally dominated by the other models; second, the APARCH and the EGARCH models clearly yield comparable results. Hence, they will be both empirically tested.

[Table 4 about here]

[Table 5 about here]

\subsection{The option pricing implementation strategy}

We apply the methodology developed in Section 3 to price European vanilla options on the French CAC 40 and the American SP 500 indexes. The strategy to price all available call options at a given date $t$ between January 2, 2006 and October 26, 2007 unfolds as follows.

1. We first select a subsample containing 4000 working days and ending on the date $t^{3}$

2. Using this subsample, we estimate the GARCH-type models (23) and (24) with GH innovations (1) favoring a two-stages estimation procedure explained in detail below. This estimation step also yields the conditional variance of returns for the date $t+1$, $h_{t+1}$.

3. Starting from the date $t+1$, we simulate sampled paths, under the risk neutral distribution:

(a) Start from the estimated conditional variance $h_{t+1}$.

(b) Compute $\theta_{t+1}^{q}$ and $\xi_{t+1}^{q}$ by solving equation (13).

(c) Sample $z_{t+1}$ from a $G H\left(\alpha, \beta+\sqrt{h_{t+1}} \theta_{t+1}^{q}, \delta, \mu\right)$ distribution using the methodology of Bibby and Sorensen (2003).

(d) Compute the log-return $Y_{t+1}$ and the conditional variance $h_{t+2}$.

(e) Then go back to step (a), replacing $t$ by $t+1$, until $t=T-1$, where $T$ is the maturity of the option we need to price.

\footnotetext{
${ }^{3}$ We compared the empirical results for sample of sizes of 3500 and 4500 , and the results work broadly the same way.
} 
This simulation scheme gives the sample returns under the properly-chosen, risk neutral distribution, from time $t$ to time $T$. The final price for the underlying asset at time $T$ is given by:

$$
S_{T}=S_{t} \prod_{k=t+1}^{T} e^{Y_{k}}
$$

4. Finally, to price a vanilla call option with time to maturity $T-t$ and strike price $K$, we simulate $N$ paths for the underlying future price $S_{T}$. The $i^{t h}$ sampled final price for the underlying process is denoted by $S_{T, i}$. Then using the standard Monte Carlo option pricing approach, we get the approximated option price $\hat{C}($.$) , as the sample average of$ the simulated final prices:

$$
\hat{C}(t, T, K)=e^{-r(T-t)} \frac{1}{N} \sum_{i=1}^{N}\left(S_{T, i}-K\right)_{+} .
$$

In practice, the number of sampled paths $N$ is equal to $10000{ }^{4}$

The previous option pricing strategy requires a few remarks. At Step (2), a Quasi Maximum Likelihood Estimation is first used to determine the parameters of the conditional variance structure. At the second stage, since we know exactly the form of the density function of a GH distribution (1) we adopt a classical maximum likelihood approach to estimate the unknown remaining parameters $(\lambda, \alpha, \beta, \delta, \mu)$ using the residuals obtained at the previous stage. The maximization of the log-likelihood of the GARCH-type processes is initialized using the unconditional variance.

The option pricing methodology used here focuses on out-of-sample option pricing errors: for a given current date, we estimate the time series parameters using a data set of constant size ending on the current date. We used 4000 observations each time. The key point in our approach is to maintain as many outliers in the data set as possible: these extreme events are essential to fit the GH parameters and to control the tail behaviors.

It is essential to note that the Monte Carlo simulation we used is indeed path-dependent: for any date $t$, we need to solve equation (13) to obtain $\theta_{t+1}^{q}$ and $\xi_{t+1}^{q}$, given the simulated volatility $h_{t+1}$ and the GH parameters.

Finally, in order to reduce the option price errors linked to the use of Monte Carlo methods, we follow the Duan and Simonato (1998) method that imposes martingality within the sampled processes. This approach makes it possible to reduce significantly the variance of the estimator of the option price.

\footnotetext{
${ }^{4}$ We compared the convergence of the estimator of the option price using 10000,15000 and 20000 simulations, and the results are globally the same. For the sake of numerical feasibility, we favored 10000 simulations.
} 
For any available date $t$ between January 2, 2006 and October 26, 2007, we reproduce the previous option pricing methodology. Thus in fact we use a rolling window estimator for both the conditional variance and GH parameters. So, we are able to check for the stability of the estimations across our data sets. Tables 6,7 and 8 provide respectively descriptive statistics for the estimated parameters of the EGARCH and APARCH models and the GH distribution. The estimated parameters for the EGARCH and APARCH models are quite stable over the different rolling windows. This is not exactly the case for the GH parameters: the standard deviation associated with each of the estimated parameters can be large. This is not a real problem for our option pricing framework, given the good pricing performances of our approach, when compared to market quotes.

[Table 6 about here]

[Table 7 about here]

[Table 8 about here]

Finally, to compare various option pricing models, we use the criterion introduced in Heston and Nandi (2000): it corresponds to the average absolute relative pricing error criterion for the working days $t$ between January 2, $2006\left(\tau_{1}\right)$ and October 26, $2007\left(\tau_{2}\right)$. Let $\hat{C}\left(t, T_{j}, K_{i}\right)$ be the estimated call option price with a time to maturity equal to $T_{j}-t$ and a strike price worth $K_{i}$. Let $C\left(t, T_{j}, K_{i}\right)$ be the corresponding quoted market option price. Then the criterion we use here is

$$
A A R P E=\frac{1}{M} \sum_{t=\tau_{1}}^{\tau_{2}} \sum_{j=1}^{J_{t}} \sum_{i=1}^{G_{t, j}}\left|\frac{\hat{C}\left(t, T_{j}, K_{i}\right)-C\left(t, T_{j}, K_{i}\right)}{C\left(t, T_{j}, K_{i}\right)}\right|,
$$

where $M$ is the total number of option prices involved in the computation of the criterion, $J_{t}$ is the number of call option maturities $T_{j}$ available at time $t$, and $G_{t, j}$ the number of strikes $K_{i}$ available at time $t$ for this particular maturity $T_{j}$.

We chose this criterion for two reasons. First, it is one of the usual criterion selected in the empirical literature. Second, this criterion is robust to the well known fact that option pricing errors are proportional to the moneyness: out of the money call option prices are very low, and so are the usual errors found. The converse is true for deep in the money option prices. This criterion rescales the errors using the level of the market option price: it is therefore robust to this effect and the analysis is made more easier.

\subsection{Discussion}

Here, we present the pricing error results obtained with our methodology and the data sets presented previously. We classically compare the EGARCH-GH and APARCH-GH models with natural competitors for which the stochastic discount factor is also constrained to be an exponential affine function of the log-returns. Through this study we want to highlight the importance of the choice of the GH distribution to model stock price processes. 
More precisely, we compare the pricing performances of the models to the Black and Scholes model (1973) (BS), the Heston and Nandi model (2000) (HN) and also to EGARCH and APARCH models with a mixture of two normal distributions (EGARCH-MN and APARCHMN) combined with an Esscher transform. Each model is estimated on an equal basis to make the inter-model comparison easier. In Table 9, we present the estimated parameters for the Heston and Nandi model obtained using the classical maximum likelihood estimators in this Gaussian framework. Table 10 provides the estimated parameters for the mixture of the two normal distributions. They have been obtained using the two stages estimation procedure already described in GH setting.

[Table 9 about here]

[Table 10 about here]

First, the choice of the previous competitors is natural because, in each of them, the exponential affine form of the pricing kernel is assumed to move from the historical distribution to the risk neutral one. Moreover, it is well known that the historical Black and Scholes (1973) model always appears as a benchmark in the financial literature and that the Heston and Nandi (2000) model is the discrete time counterpart of one of the main models used in the banking industry for option pricing: the Heston (1993) stochastic volatility model. Finally, in the EGARCH-MN and APARCH-MN models, the use of a mixture of two normal distributions to model the innovations may be interestingly compared to our choice of the GH distribution, because these two families have exactly the same number of parameters.

Tables 11 and 12 present the values of the AARPE criterion given in (29) for each model.

[Table 11 about here]

[Table 12 about here]

The previous tables yield the following empirical results:

- In the CAC 40 case, there are four different conclusions that can be raised. First, the Black Scholes and the Heston and Nandi models are clearly lagging behind, as they fail to produce pricing errors as low as their competitors. However, we used them as a benchmark, allowing us to compare the results obtained with the model proposed here to the usual option pricing models. Second, when it comes to selecting a particular volatility structure, the EGARCH strikingly outperforms the the APARCH model, whatever the conditional distribution used to compute the theoretical option prices. This is true regardless of the moneyness and maturity selected, but for two cases: in the case of a moneyness below 0.8 and a maturity between 0.25 and 0.5 years or superior to one year. Third, the conditional distribution providing the lowest errors is the Generalized Hyperbolic distribution: this is true whatever the volatility structure, the moneyness and the maturity considered. Hence, all in all, the EGARCH-GH is the model providing the best results. The fourth conclusion is the most important of our work: the scale of the errors obtained with the methodology used here is globally low. 
For example, for maturities between six months and one year, the errors range between $5 \%$ and $70 \%$ broadly speaking. Unlike Barone-Adesi et al. (2008), these errors are obtained without using any parameter optimization used to fit as well as possible the option prices. When optimizing the volatility parameters, they obtain errors ranging from $3 \%$ to $40 \%$, broadly speaking again.

- The SP 500 case yields fairly comparable results. Again, the first one is related to the inability of either the Black Scholes model or the Heston and Nandi one to provide mispricing errors as close to zero as the remaining competitors. When comparing the performances of EGARCH models vs. APARCH models, a more balanced picture can be drawn than in the CAC 40 case: the EGARCH modeling provides superior performances for out-of-the-money options. In-the-money options prices are better approximated using an APARCH volatility structure: the non-linearity of the APARCH model seems to serve the purpose of option pricing in the case of strikes below the current value of the spot price of the underlying asset. When considering the conditional distribution to be used under the risk neutral distribution, the GH distribution provides the lowest pricing errors.

Thus, the main findings of this empirical study can be summarized as follow. First, the models based on a fine understanding of the tail behaviors - i.e. those based on the GH and the MN distributions - yield the best possible results. Second, our models based on the GH distribution outperform most of the other models for a wide range of moneynesses and maturities. This is especially true for the right tail of the distribution: deep out-of-the-money option prices are fairly well approximated with an EGARCH-GH model, once compared to the performance of the usual competitors. Again, the overall sizes of the errors obtained here are close to what is obtained when calibrating the parameters to fit the option prices as good as possible: starting from a statistically realistic historical distribution, we impose no arbitrage restrictions based on a specific choice about the shape of risk aversion. Then, we compute the option prices, using an enhanced Monte Carlo method. This approach is less time consuming than the "direct parameter calibration" one, as it avoids the Monte Carlo based optimization. The key lesson here seems to be that a successful option pricing approach should focus on the tail behavior of the returns rather than on the dynamics of volatility. This empirical observation is consistent both under the risk neutral and under the historical distribution: this is at least the conclusion that can be raised from the observation of the Amisano and Giacomini (2007) test presented previously. Hence, when it comes to returns' distribution forecast - risk neutral or historical - the volatility dynamics should not be the primary issue to focus on.

\section{Conclusion}

In this paper, we presented new models within the discrete time GARCH model class, based on two assumptions. First, the log-returns of the underlying asset are conditionally generalized hyperbolically distributed. Then, to price vanilla options, we choose the risk-neutral 
measure given by the Esscher transform that is equivalent to supposing an exponential affine form for the stochastic discount factor. Using these assumptions, we show how to price options using Monte Carlo methods and compare the empirical performances of our model with the one found in the existing literature. The performances of the model are obtained to be close to those found when performing a calibration exercise.

To conclude, let us mention that this work can be extended considering other risk-neutralized frameworks for the generalized hyperbolic distribution, using for instance the extended Girsanov principle introduced by Elliot and Madan (1998), or the martingalized historical approach presented in Chorro et al. (2009).

\section{References}

[1] Abramowitz, M. and Stegun, I. (1964). Handbook of mathematical functions with formulas, graphs and mathematical tables. Dover: New-York.

[2] Amisano, G. and Giacomini, R., (2007). Comparing Density Forecasts viaWeighted Likelihood Ratio Tests. Journal of Business \& Economic Statistics, 25, 77-190.

[3] Alexander, C. and Lazar, E., (2006). Normal mixture GARCH(1,1): Applications to exchange rate modelling. Journal of Applied Econometrics, 21, 307-336.

[4] Barndorff-Nielsen, OE., (1977). Exponentially decreasing distributions for the logarithm of particle size. Proceedings of the Royal Society of London Series A, 353, 401-419.

[5] Barndorff-Nielsen, OE., (1995). Normal inverse Gaussian processes and the modelling of stock returns. Research Report 300, Department Theoretical Statistics, Aarhus University.

[6] Barndorff-Nielsen, O.E. and Blaesild, P., (1981). Hyperbolic Distributions and Ramifications: Contributions to Theory and Applications. In: Taillie C, Patil GP, Baldessari BA (Eds), Statistical distributions in scientific work, vol.4. Reidel, Dordrecht, 19-44.

[7] Barone-Adesi, G., Engle, R.F. and Mancini L.A., (2008). GARCH Option Pricing Model in Incomplete Markets. Review of Financial Studies, 21(3), 1223-1258.

[8] Bibby, B.M. and Sorensen, M., (2003). Hyperbolic processes in Finance. In: Rachev ST (Ed), Handbook of Heavy Tailed Distributions in Finance. Elsevier Science, 212-248.

[9] Black, F. and Scholes, M., (1973). The pricing of Options and corporate liabilities. Journal of political Economy, 81, 637-659.

[10] Blaesild, P., (1981). The Two-Dimensional Hyperbolic Distribution and Related Distributions with an Application to Johannsen's Bean Data. Biometrika, 1 (68), 251-263.

[11] Bollerslev, T., (1986). Generalized Autoregressive Conditional Heteroskedasticity. Journal of Econometrics, 31, 307-327. 
[12] Bouchaud, JP. and Potters, M., (2003). Theory of financial risk and derivative pricing: from statistical physics to risk management. Cambridge University Press.

[13] Bühlmann, H., Delbaen, F., Embrechts, P. and Shiryaev, AN., (1996). No-Arbitrage Change of Measure and Conditional Esscher Transforms. CWI Quarterly, 9, 291-317.

[14] Christoffersen, P., Heston, S.L. and Jacobs, K., (2006). Option valuation with conditional skewness. Journal of Econometrics, 131, 253-284.

[15] Chorro, C., Guégan, D. and Ielpo, F., (2010). Martingalized historical approach for option pricing. Finance Research Letters, 7(1), 24-28.

[16] Cochrane, J., (2001). Asset Pricing. Princeton University Press.

[17] Diebold, F. X. and Mariano, R. S., (1995). Comparing predictive accuracy. Journal of Business \& Economic Statistics, 13(3), 253-63.

[18] Ding Z., Granger, C.W.J. and Engle, R.F., (1993). A long memory property of stock market returns and a new model. Journal of Empirical Finance, 1, 83-106.

[19] Duan, J.C., (1995). The GARCH Option Pricing Model. Mathematical Finance, 5, 13-32.

[20] Duan, J.C. and Simonato, J.G., (1998). Empirical Martingale Simulation for asset Prices. Management Science, 44(9), 1218-1233.

[21] Eberlein, E. and Prause, K., (2002). The generalized hyperbolic model: financial derivatives and risk measures. In: Geman H, Madan D, Pliska S, Vorst T (Eds), Mathematical Finance-Bachelier Congress 2000, Springer Verlag, 245-267.

[22] Elliott, R. and Madan, D., (1998). A Discrete Time Equivalent Martingale Measure. Mathematical Finance, 2(8), 127-152.

[23] Embretchs, P., Frey, R. and McNeil, A.J., (2005). Quantitative Risk Management. Princeton Univ. Press.

[24] Engle, R., (1982). Autoregressive Conditional Heteroskedasticity with Estimates of the variance of U.K. inflation, Econometrica, 50, 987-1008.

[25] Esscher, F., (1932). On the probability function in the collective theory of risk. Skandinavisk Aktuarietidskrift, 15, 175 -195.

[26] Fôllmer, H. and Schweiser, M., (1991). Hedging of Contingent Claims under Incomplete Information. In: Davis MHA, Elliot RJ (Eds), Applied Stochastic Analysis, Gordon and Breach: London, 389-414.

[27] Gerber, H.U. and Shiu, S.W., (1994a). Martingale Approach to Pricing Perpetual American Options. In: Proceedings of the 4th AFIR International Colloqium, Transaction of Society of Actuaries: Orlando, 659-689. 
[28] Gerber, H.U. and Shiu, S.W., (1994b). Option Pricing by Esscher Transforms. Transaction of Society of Actuaries, 46, 99-191.

[29] Glosten, L.R., Jagannathan, R. and Runkle, D.E., (1993). On the relation between the expected value and the volatility of the nominal excess return on stocks. Journal of Finance, 48(5), 1779-1801.

[30] Guégan, D. and Zhang, J., (2009). Pricing Bivariate Option under GARCH-GH Model with Dynamic Copula: Application for Chinese Market. The European Journal of finance (in press).

[31] Heston, S.L., (1993). A closed-form solution for options with stochastic volatility, with applications to bond and currency options. The Review of Financial Studies, 6, 327-343.

[32] Heston, S.L. and Nandi, S., (2000). A Closed-Form GARCH Option Valuation. The Review of Financial Studies, 13, 585-625.

[33] Mainardi, F. and Rogosin, S., (2006). The Origin of Infinitely Divisible Distributions: from de Finetti's Problem to Lévy-Khintchine Formula. Mathematical Methods in Economics and Finance, 1, 37-55.

[34] Nelson C.R. and Siegel A.F., (1987). Parsimonious modeling of yield curves. Journal of Business, 59, 473-489.

[35] Nelson, D.B., (1991). Conditional Heteroskedasticity in Asset Returns. Econometrica, $59,347-370$.

[36] Rubinstein, M., (1978). The Strong Case for the Generalized Logarithmic Utility Model as the Premier Model of Financial Markets. Journal of Finance, 31, 551-557.

[37] Siu, T.K., Tong, H. and Yang, H., (2004). On Pricing Derivatives under GARCH models: A Dynamic Gerber-Shiu Approach. North American Actuarial Journal, 8, 17-31.

[38] Svensson, L.E.O., (1994). Estimating and Interpreting Forward Interest Rates: Sweden 1992-1994. Working Paper No. WP/94/I 14, International Monetary Fund: Washington D.C. 


\section{Appendix: Proofs}

\section{Proof of Proposition 2}

Here we provide the proof of the Proposition 2.

Proof: First, when $s \leq T$, then for an $\mathcal{F}_{s}$ measurable and non-negative random variable $Z$ we may deduce from the martingale property of $\left(L_{t}\right)$ that

$$
E_{\mathbb{Q}}[Z]=E_{\mathbb{P}}\left[L_{s} Z\right] .
$$

Moreover, for $T \geq t \geq s>0$, if $Z$ (resp. $X$ ) is $\mathcal{F}_{s}$ (resp. $\mathcal{F}_{t}$ ) measurable and non-negative then

$$
E_{\mathbb{Q}}[X Z]=E_{\mathbb{P}}\left[L_{T} X Z\right]=E_{\mathbb{P}}\left[E_{\mathbb{P}}\left[X L_{T} \mid \mathcal{F}_{s}\right] Z\right]=E_{\mathbb{P}}\left[E_{\mathbb{P}}\left[X E_{\mathbb{P}}\left[L_{T} \mid \mathcal{F}_{t}\right] \mid \mathcal{F}_{s}\right] Z\right]
$$

thus

$$
E_{\mathbb{Q}}[X Z]=E_{\mathbb{P}}\left[E_{\mathbb{P}}\left[L_{t} X \mid \mathcal{F}_{s}\right] Z\right]=E_{\mathbb{P}}\left[\frac{L_{s}}{L_{s}} E_{\mathbb{P}}\left[X L_{t} \mid \mathcal{F}_{s}\right] Z\right]=E_{\mathbb{Q}}\left[\frac{1}{L_{s}} E_{\mathbb{P}}\left[X L_{t} \mid \mathcal{F}_{s}\right] Z\right]
$$

Hence,

$$
E_{\mathbb{Q}}\left[X \mid \mathcal{F}_{s}\right]=\frac{1}{L_{s}} E_{\mathbb{P}}\left[X L_{t} \mid \mathcal{F}_{s}\right]
$$

a) and b) easily follow

\section{Proof of Proposition 3}

Here we provide the proof of the Proposition 3.

Proof: From the Kolmogorov representation theorem (see e.g Mainardi and Rogosin, 2006 for an interesting historical approach to this result), we have for all $u \in \mathcal{D}_{\mathbb{G}_{t-1}}$,

$$
\log \left(\mathbb{G}_{t-1}(u)\right)=\gamma_{t} u+\int_{-\infty}^{+\infty}\left(e^{z u}-1-z u\right) \frac{d K_{t}(z)}{z^{2}}
$$

where $\gamma_{t}$ is an $\mathcal{F}_{t-1}$ measurable real value random variable and $K_{t}$ an $\mathcal{F}_{t-1}$ measurable random variable with values in the space of the non-decreasing and bounded functions with limit zero in $-\infty$. Thus, from Proposition $2, \forall u \in\left\{\theta \in \mathbb{R} ; \theta+\theta_{t}^{q} \in \mathcal{D}_{\mathbb{G}_{t-1}}\right\}$,

$$
\log \left(E_{\mathbb{Q}}\left[e^{u Y_{t}} \mid \mathcal{F}_{t-1}\right]\right)=\gamma_{t} u+\int_{-\infty}^{+\infty}\left(e^{z\left(u+\theta_{t}^{q}\right)}-e^{\theta_{t}^{q} z}-z u\right) \frac{d K_{t}(z)}{z^{2}}
$$

thus

$$
\log \left(E_{\mathbb{Q}}\left[e^{u Y_{t}} \mid \mathcal{F}_{t-1}\right]\right)=\tilde{\gamma}_{t} u+\int_{-\infty}^{+\infty}\left(e^{z u}-1-u z\right) \frac{e^{\theta_{t}^{q} z} d K_{t}(z)}{z^{2}}
$$

where

$$
\tilde{\gamma}_{t}=\gamma_{t}+\int_{-\infty}^{+\infty}\left(e^{\theta_{t}^{q} z}-1\right) z \frac{d K_{t}(z)}{z^{2}} .
$$

Since $\mathbb{G}_{t-1}$ is twice differentiable, we have in particular that

$$
\int_{-\infty}^{+\infty} e^{\theta_{t}^{q} z} d K_{t}(z)<\infty
$$


and we may define $\forall x \in \mathbb{R}$,

$$
\tilde{K}_{t}(x)=\int_{-\infty}^{x} e^{\theta_{t}^{q} z} d K_{t}(z)
$$

that is an $\mathcal{F}_{t-1}$ measurable random variable with values in the space of the non-decreasing and bounded functions with limit zero in $-\infty$. The conclusion follows from (31) and from the Kolmogorov representation theorem.

\section{Proof of Proposition 4}

Here we provide the proof of the Proposition 4.

Proof: For $|\beta+u|<\alpha$,

$$
\mathbb{G}_{G H}(u)=e^{\mu u}\left(\frac{\alpha^{2}-\beta^{2}}{\alpha^{2}-(\beta+u)^{2}}\right)^{\frac{\lambda}{2}} \frac{K_{\lambda}\left(\delta \sqrt{\alpha^{2}-(\beta+u)^{2}}\right)}{K_{\lambda}\left(\delta \sqrt{\alpha^{2}-\beta^{2}}\right)},
$$

hence $\mathbb{G}_{G H}$ is twice differentiable. Moreover, $\Phi(\theta)=\log \left(\frac{\mathbb{G}_{G H}(1+\theta)}{\mathbb{G}_{G H}(\theta)}\right)$ is defined on the interval ] $-(\alpha+\beta), \alpha-\beta-1\left[\right.$ that is not empty because $\alpha>\frac{1}{2}$. Thus we may apply Proposition 1 and the unicity holds. The existence needs still to be proved.

a) For $x>0$, we define $\Psi(x)=\log \left(\frac{K_{\lambda}(x)}{x^{\lambda}}\right)$. Thus,

$$
\Phi(\theta)=\mu+\Psi\left(\delta \sqrt{\alpha^{2}-(\beta+1+\theta)^{2}}\right)-\Psi\left(\delta \sqrt{\alpha^{2}-(\beta+\theta)^{2}}\right) .
$$

For the properties of the Bessel function used in the sequel we refer the reader to Abramowitz and Stegun (1964). If $\lambda>0$,

$$
\frac{K_{\lambda}(x)}{x^{\lambda}} \sim_{x \rightarrow 0+} \frac{\Gamma[\lambda] 2^{\lambda-1}}{x^{2 \lambda}} .
$$

So we have $\lim _{\theta \rightarrow \alpha-\beta-1} \Phi(\theta)=+\infty$ and $\lim _{\theta \rightarrow-(\alpha+\beta)} \Phi(\theta)=-\infty$. The conclusion follows from the intermediate value theorem. When $\lambda=0$, we may conclude as before, remembering that $K_{0}(x) \sim_{x \rightarrow 0+}-\log (x / 2)-\gamma$ where $\gamma$ is the Euler-Mascheroni constant.

b) When $\lambda<0$ using the relation $K_{\lambda}(x)=K_{-\lambda}(x)$ we obtain that $\frac{K_{\lambda}(x)}{x^{\lambda}} \sim_{x \rightarrow 0+} \Gamma[-\lambda] 2^{-\lambda-1}$. Thus, $\lim _{\theta \rightarrow \alpha-\beta-1} \Phi(\theta)=\mu+C$ and $\lim _{\theta \rightarrow-(\alpha+\beta)} \Phi(\theta)=\mu-C$ and we conclude applying again the intermediate value theorem. 


\section{Tables and Figures}

\begin{tabular}{c|c|c|c}
\hline \hline \multicolumn{4}{c}{ CAC 40 } \\
\hline \hline Number of observations & Mean & Minimum & Maximum \\
4159 & 0.0003 & -0.1014 & 0.0823 \\
\hline \hline Median & Annualized Volatility & Skewness & Kurtosis \\
0.0004 & 0.208 & -0.2862 & 4.4774 \\
\hline \hline
\end{tabular}

\begin{tabular}{c|c|c|c}
\hline \hline \multicolumn{4}{|c}{ SP 500 } \\
\hline \hline Number of observations & Mean & Minimum & Maximum \\
4159 & 0.0003 & -0.2290 & 0.0871 \\
\hline \hline Median & Annualized Volatility & Skewness & Kurtosis \\
0.0005 & 0.1700 & -2.0601 & 44.4738 \\
\hline \hline
\end{tabular}

Table 1: Descriptive statistics for the CAC 40 and the SP 500 data sets from January 2, 1988 to October 26, 2007.

\begin{tabular}{c|c|c}
\hline \hline & CAC 40 & SP 500 \\
\hline \hline KS p-value for NIG & 0.3 & 0.67 \\
KS p-value for HYP & 0.73 & 0.69 \\
KS p-value for GH & 0.31 & 0.78 \\
KS p-value for Normal & 0 & 0 \\
\hline \hline AD p-value for NIG & 0.45 & 0.5 \\
AD p-value for HYP & 0.5 & 0.47 \\
AD p-value for GH & 0.23 & 0.55 \\
AD p-value for Normal & 0 & 0 \\
\hline \hline
\end{tabular}

Table 2: Kolmogorov-Smirnov (KS) and Andersen-Darling (AD) adequation tests for CAC 40 and SP 500 standardized residuals.

This table presents the Kolmogorov-Smirnov and Andersen-Darling adequation tests, testing the adequation of the NIG, Hyperbolic, Generalized Hyperbolic (definitions are given in Subsection 2.2) and Normal distributions to a data set of the daily log-returns of each index. Time varying variance has been filtered out using an EGARCH process. The samples start on January 2, 1988 and end on October 26, 2007. 


\begin{tabular}{|c|c|c|c|c|c|c|}
\hline \multicolumn{7}{|c|}{ CAC 40} \\
\hline \multicolumn{7}{|c|}{ Number of available option contracts } \\
\hline & $<<.8$ & {$[.8-.9]$} & {$[.9-1]$} & {$[1-1.1]$} & {$[1.1-1.2]$} & $>1.2$ \\
\hline $.25<$ Maturity $<.5$ & 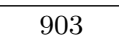 & 2579 & 2879 & 2835 & 1519 & 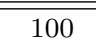 \\
\hline $.5<$ Maturity $<1$ & 1294 & 4577 & 5530 & 5511 & 4065 & 1859 \\
\hline Maturity $>1$ & 1026 & 7262 & 15067 & 16010 & 14823 & 14688 \\
\hline \multicolumn{7}{|c|}{ Average option price } \\
\hline Maturity/Moneyness & $\overline{<<.8}$ & {$[.8-.9]$} & {$[\cdot .9-1]$} & [1-1.1] & {$[1.1-1.2]$} & $>1.2$ \\
\hline 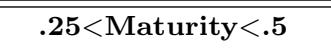 & 1435.28 & 949.24 & 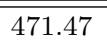 & 139.33 & 20.60 & 2.42 \\
\hline $.5<$ Maturity $<1$ & 1547.91 & 1051.23 & 621.27 & 287.88 & 94.31 & 19.53 \\
\hline Maturity $>1$ & 1651.09 & 1158.02 & 780.15 & 483.01 & 261.69 & 124.69 \\
\hline
\end{tabular}

\begin{tabular}{|c|c|c|c|c|c|c|}
\hline \multicolumn{7}{|c|}{ SP 500} \\
\hline \multicolumn{7}{|c|}{ Number of available option contracts } \\
\hline & $<<.8$ & {$[.8-.9]$} & {$[.9-1]$} & {$[1-1.1]$} & {$[1.1-1.2]$} & $>1.2$ \\
\hline $.25<$ Maturity $<.5$ & 660 & 1918 & 2290 & 2509 & 854 & 0 \\
\hline $.5<$ Maturity $<1$ & 422 & 1898 & 4212 & 5393 & 3874 & 779 \\
\hline Maturity $>1$ & 124 & 1218 & 4310 & 6353 & 5056 & 3955 \\
\hline \multicolumn{7}{|c|}{ Average option price } \\
\hline Maturity/Moneyness & $<.8$ & {$[.8-.9]$} & {$[.9-1]$} & [1-1.1] & {$[1.1-1.2]$} & $>1.2$ \\
\hline $.25<$ Maturity $<.5$ & 348.47 & 247.59 & 131.22 & 37.28 & 6.16 & - \\
\hline $.5<$ Maturity $<1$ & 346.34 & 270.76 & 165.48 & 83.91 & 27.32 & 5.29 \\
\hline Maturity $>1$ & 414 & 283.10 & 220.36 & 154.73 & 96.98 & 43.96 \\
\hline
\end{tabular}

Table 3: Descriptive statistics for the option data sets used in the paper. The option data sets start on January 2, 2006 and end on October 26, 2007.

\begin{tabular}{cccc}
\hline \hline & EGARCH & APARCH & GJR \\
\hline \hline EGARCH & - & 0.66 & 2.16 \\
APARCH & - & - & 2.43 \\
\hline \hline
\end{tabular}

Table 4: Amisano and Giacomini (2007)'s test comparing the different GARCH models insample. The results are based on the CAC 40 returns from January 2, 1988 to October 26, 2007. Example: when comparing whether the EGARCH is better than the GJR model in the Amisano and Giacomini (2007)'s sense, the statistic obtained is 2.16: this means that the EGARCH statistically dominates the GJR model at a $5 \%$ threshold.

\begin{tabular}{cccc}
\hline \hline & EGARCH & APARCH & GJR \\
\hline \hline EGARCH & - & -0.17 & 2.74 \\
APARCH & - & - & 2.70 \\
\hline \hline
\end{tabular}

Table 5: Amisano and Giacomini (2007)'s test comparing the different GARCH models insample. The results are based on the SP 500 returns from January 2, 1988 to October 26, 2007. Example: when comparing whether the EGARCH is better than the GJR model in the Amisano and Giacomini (2007)'s sense, the statistic obtained is 2.74: this means that the EGARCH statistically dominates the GJR model at a $5 \%$ threshold. 


\begin{tabular}{ccccccc}
\hline \hline Index & & $a_{0}$ & $b_{1}$ & $a_{1}$ & $\gamma$ & $\lambda_{0}$ \\
\hline \hline CAC 40 & Average & -5.51 & 0.47 & 0.01 & 0.91 & 0.08 \\
& Std. Dev. & 0.33 & 0.04 & 0.02 & 0.02 & 0.03 \\
SP 500 & Average & -5.22 & 0.55 & 0.005 & 0.91 & 0.15 \\
& Std. Dev. & 1.36 & 0.13 & 0.05 & 0.12 & 0.06 \\
\hline \hline
\end{tabular}

Table 6: Parameter estimates of the EGARCH model estimated on each working day from January 2, 2006 to October 26, 2007 using Quasi-maximum likelihood and 4000 log-returns.

\begin{tabular}{cccccccc}
\hline \hline Index & & $w$ & $a$ & $\gamma$ & $\Delta$ & $b$ & $\lambda_{0}$ \\
\hline \hline CAC 40 & Average & $2.86 \mathrm{E}-4$ & 0.34 & 0.15 & 1.29 & 0.48 & 0.21 \\
& Std. Dev. & $7.62 \mathrm{E}-4$ & 0.19 & 0.24 & 0.58 & 0.30 & 0.29 \\
\multirow{3}{*}{ SP 500 } & Average & $5.54 \mathrm{E}-4$ & 0.43 & 0.11 & 1.65 & 0.23 & 0.25 \\
& Std. Dev. & $1.64 \mathrm{E}-4$ & 0.20 & 0.21 & 0.41 & 0.28 & 0.30 \\
\hline \hline
\end{tabular}

Table 7: Parameter estimates of the APARCH model estimated on each working day from January 2, 2006 to October 26, 2007 using Quasi-maximum likelihood and 4000 log-returns.

\begin{tabular}{cccccccc}
\hline \hline Index & Model & & $\alpha$ & $\beta$ & $\delta$ & $\mu$ & $\lambda$ \\
\hline \hline CAC 40 & \multirow{2}{*}{ EGARCH } & Average & 308.42 & -2.77 & 335.64 & 3.04 & -78.46 \\
& & Std. Dev. & 139.90 & 5.08 & 152.02 & 5.51 & 76.68 \\
\multirow{2}{*}{ CAC 40 } & \multirow{2}{*}{ APARCH } & Average & 219.46 & 4.10 & 261.86 & -0.72 & -26.62 \\
& & Std. Dev. & 149.04 & 76.89 & 213.83 & 4.62 & 42.35 \\
\multirow{2}{*}{ SP 500 } & \multirow{2}{*}{ EGARCH } & Average & 357.14 & 0.48 & 376.49 & -0.76 & -12.70 \\
& & Std. Dev. & 161.49 & 4.87 & 172.91 & 4.20 & 22.13 \\
SP 500 & \multirow{2}{*}{ APARCH } & Average & 154.69 & 13.44 & 195.50 & -0.77 & -21.73 \\
& & Std. Dev. & 139.26 & 46.67 & 205.04 & 3.74 & 41.08 \\
\hline \hline
\end{tabular}

Table 8: Parameter estimates of the GH distribution estimated on each working day from January 2, 2006 to October 26, 2007 using the 2 stages estimation procedure and 4000 logreturns. 


\begin{tabular}{ccccccc}
\hline \hline Index & & $\omega$ & $\beta$ & $\alpha$ & $\gamma$ & $\lambda_{0}$ \\
\hline \hline \multirow{2}{*}{ CAC40 } & Average & $5.09 \mathrm{E}-06$ & 0.86 & $2.22 \mathrm{E}-05$ & 6.72 & -0.33 \\
& St. Dev. & $2.58 \mathrm{E}-03$ & 20.50 & $1.99 \mathrm{E}-03$ & 384.81 & 13.58 \\
\multirow{3}{*}{ SP500 } & Average & $2.89 \mathrm{E}-05$ & 0.39 & $7.85 \mathrm{E}-06$ & 1.67 & -0.06 \\
& St. Dev. & $2.53 \mathrm{E}-05$ & 0.44 & $1.21 \mathrm{E}-05$ & 2.63 & 0.15 \\
\hline \hline
\end{tabular}

Table 9: Parameter estimates of the the HN model estimated on each working day from January 2, 2006 to October 26, 2007 using maximum likelihood and 4000 log-returns. We suppose here that $Y_{t}=r+\lambda_{0} h_{t}+\sqrt{h_{t}} z_{t}$, where the $z_{t}$ 's are i.i.d $\mathcal{N}(0,1)$ and with a structure for the conditional variance given by $h_{t}=\omega+\beta h_{t-1}+\alpha\left(z_{t-1}-\gamma \sqrt{h_{t-1}}\right)^{2}$. The restriction $\beta+\alpha \gamma^{2}<1$ is imposed to ensure process stationary, with finite mean and variance.

\begin{tabular}{cccccccc}
\hline \hline Index & Model & & $\phi$ & $\mu_{1}$ & $\mu_{2}$ & $\sigma_{1}$ & $\sigma_{2}$ \\
\hline \hline \multirow{2}{*}{ CAC 40 } & \multirow{2}{*}{ EGARCH } & Average & 0.62 & 0.97 & -0.87 & 0.47 & 0.49 \\
& & Std. Dev. & 0.01 & 0.11 & 0.10 & 0.07 & 0.12 \\
CAC 40 & \multirow{2}{*}{ APARCH } & Average & 0.62 & -0.24 & 0.10 & 0.66 & 0.65 \\
& & Std. Dev. & 0.04 & 0.92 & 0.89 & 0.23 & 0.27 \\
\multirow{2}{*}{ SP 500 } & \multirow{2}{*}{ EGARCH } & Average & 0.63 & -0.12 & 0.09 & -0.58 & 0.47 \\
& & Std. Dev. & 0.03 & 0.87 & 0.89 & 0.17 & 0.17 \\
SP 500 & \multirow{2}{*}{ APARCH } & Average & 0.62 & -0.50 & 0.10 & 0.60 & 0.58 \\
& & Std. Dev. & 0.05 & 0.93 & 1.11 & 0.36 & 0.34 \\
\hline \hline
\end{tabular}

Table 10: Parameter estimates of the mixture of the two normal distributions estimated on each working day from January 2, 2006 to October 26, 2007 using the 2 stages estimation procedure and 4000 log-returns: we suppose in this case that the density of the $z_{t}$ 's is given by $p(x)=\phi n\left(x, \mu_{1}, \sigma_{1}^{2}\right)+(1-\phi) n\left(x, \mu_{2}, \sigma_{2}^{2}\right)$ where $n\left(x, \mu, \sigma^{2}\right)$ is the density of a $\mathcal{N}\left(\mu, \sigma^{2}\right)$. 


\begin{tabular}{|c|c|c|c|c|c|c|}
\hline \multicolumn{7}{|c|}{$.25<$ Maturity $<.5$} \\
\hline Moneyness & $<.8$ & {$[.8-.9]$} & {$[.9-1]$} & {$[1-1.1]$} & [1.1-1.2] & $>1.2$ \\
\hline BS & 0.049 & 0.073 & 0.234 & 1.694 & 20.504 & 56.189 \\
\hline HN & 0.043 & 0.069 & 0.219 & 1.560 & 25.422 & 73.120 \\
\hline EGARCH-MN & 0.055 & 0.077 & 0.174 & 0.656 & 6.604 & 55.893 \\
\hline EGARCH-GH & 0.046 & 0.058 & 0.118 & 0.294 & 0.989 & 1.492 \\
\hline APARCH-MN & 0.053 & 0.084 & 0.189 & 1.234 & 21.655 & 57.764 \\
\hline APARCH-GH & 0.046 & 0.063 & 0.126 & 0.553 & 3.243 & 4.895 \\
\hline \multicolumn{7}{|c|}{$.5<$ Maturity $<1$} \\
\hline Mon & $<.8$ & {$[.8-.9]$} & {$[.9-1]$} & [1-1.1] & [1.1-1.2] & $>1.2$ \\
\hline BS & 0.067 & 0.122 & 0.252 & 0.749 & 18 & 9.852 \\
\hline HN & 0.057 & 0.120 & 0.248 & 0.766 & 3.143 & 9.615 \\
\hline EGARCH-MN & 0.069 & 0.101 & 0.152 & 0.384 & 1.245 & 4.542 \\
\hline EGARCH-GH & 0.055 & 0.071 & 0.080 & 0.181 & 0.343 & 0.699 \\
\hline APARCH-MN & 0.073 & 0.123 & 0.139 & 0.557 & 2.127 & 7.542 \\
\hline APARCH-GH & 0.058 & 0.081 & 0.100 & 0.278 & 0.743 & 3.802 \\
\hline \multicolumn{7}{|c|}{ Maturity $>1$} \\
\hline Moneyness & $<.8$ & {$[.8-.9]$} & {$[.9-1]$} & [1-1.1] & [1.1-1.2] & $>1.2$ \\
\hline BS & 0.132 & 0.215 & 0.361 & 0.664 & 1.379 & 3.064 \\
\hline $\mathbf{H N}$ & 0.139 & 0.220 & 0.387 & 0.727 & 1.525 & 3.549 \\
\hline EGARCH-MN & 0.125 & 0.150 & 0.177 & 0.272 & 0.534 & 1.287 \\
\hline EGARCH-GH & 0.106 & 0.111 & 0.088 & 0.104 & 0.169 & 0.307 \\
\hline APARCH-MN & 0.119 & 0.168 & 0.194 & 0.459 & 1.282 & 2.876 \\
\hline APARCH-GH & 0.104 & 0.124 & 0.137 & 0.221 & 0.454 & 1.124 \\
\hline
\end{tabular}

Table 11: Absolute average pricing errors for CAC 40 option prices disaggregated by maturities and moneyness. 


\begin{tabular}{|c|c|c|c|c|c|c|}
\hline \multicolumn{7}{|c|}{$.25<$ Maturity $<.5$} \\
\hline Moneyness & $<.8$ & {$[.8-.9]$} & {$[.9-1]$} & [1-1.1] & {$[1.1-1.2]$} & $>1.2$ \\
\hline BS & 0.161 & 0.142 & 0.110 & 0.595 & 3.605 & \\
\hline $\mathrm{HN}$ & 0.162 & 0.145 & 0.108 & 0.590 & 3.406 & \\
\hline EGARCH-MN & 0.158 & 0.143 & 0.114 & 0.582 & 1.115 & \\
\hline EGARCH-GH & 0.162 & 0.144 & 0.106 & 0.546 & 0.851 & \\
\hline APARCH-MN & 0.145 & 0.129 & 0.131 & 0.634 & 2.912 & \\
\hline APARCH-GH & 0.129 & 0.126 & 0.125 & 0.620 & 2.405 & \\
\hline \multicolumn{7}{|c|}{$.5<$ Maturity $<1$} \\
\hline Moneyness & $<.8$ & {$[.8-.9]$} & {$[.9-1]$} & [1-1.1] & [1.1-1.2] & $>1.2$ \\
\hline $\mathrm{BS}$ & 0.297 & 0.174 & 0.150 & 0.201 & 1.094 & 4.714 \\
\hline $\mathbf{H N}$ & 0.296 & 0.173 & 0.151 & 0.205 & 1.024 & 5.318 \\
\hline EGARCH-MN & 0.433 & 0.219 & 1.132 & 0.415 & 0.733 & 0.909 \\
\hline EGARCH-GH & 0.296 & 0.172 & 0.128 & 0.325 & 0.716 & 0.828 \\
\hline APARCH-MN & 0.357 & 0.194 & 0.156 & 0.386 & 0.873 & 2.512 \\
\hline APARCH-GH & 0.234 & 0.113 & 0.145 & 0.360 & 0.841 & 1.675 \\
\hline \multicolumn{7}{|c|}{ Maturity $>1$} \\
\hline Moneyness & $<.8$ & {$[.8-.9]$} & {$[.9-1]$} & [1-1.1] & [1.1-1.2] & $>1.2$ \\
\hline BS & 0.219 & 0.365 & 0.246 & 0.207 & 0.401 & 1.487 \\
\hline $\mathbf{H N}$ & 0.221 & 0.369 & 0.260 & 0.197 & 0.379 & 1.526 \\
\hline EGARCH-MN & 0.279 & 0.367 & 0.216 & 0.167 & 0.363 & 0.570 \\
\hline EGARCH-GH & 0.221 & 0.359 & 0.206 & 0.131 & 0.307 & 0.567 \\
\hline APARCH-MN & 0.157 & 0.358 & 0.204 & 0.267 & 0.424 & 0.973 \\
\hline APARCH-GH & 0.091 & 0.241 & 0.147 & 0.249 & 0.415 & 0.655 \\
\hline
\end{tabular}

Table 12: Absolute average pricing errors for SP 500 option prices disaggregated by maturities and moneyness. 
CAC
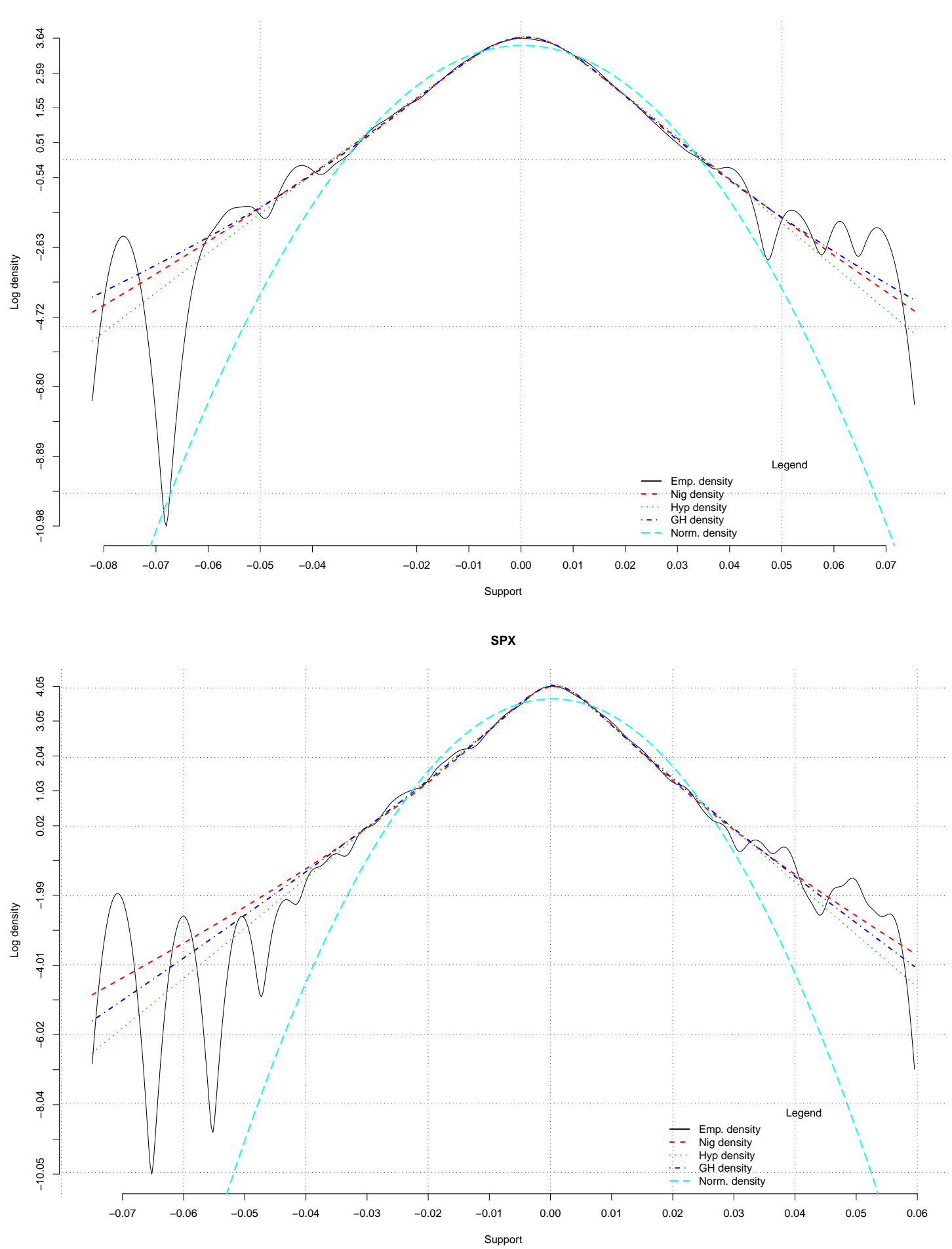

Figure 1: Log-density

This figure presents the empirical log-density (plain black line) vs. the estimated log-density obtained with the NIG (red), Hyperbolic (green), Generalized Hyperbolic (dark blue) and Normal (light blue) distributions using the CAC 40 and the SP 500 returns data sets. Time varying variance has been filtered out using an EGARCH process. The samples start on January 2, 1988 and end on October 26, 2007. 


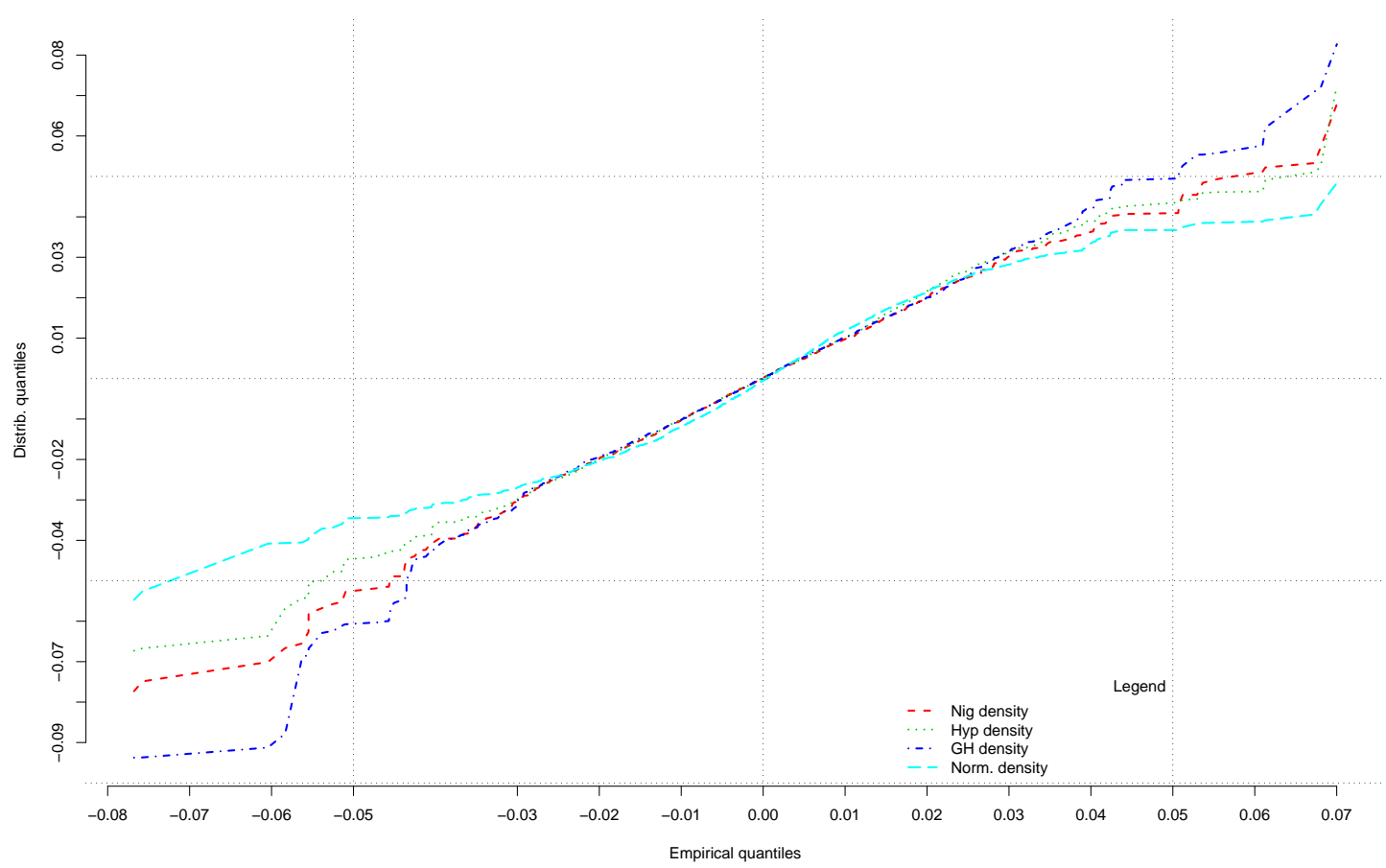

SPX

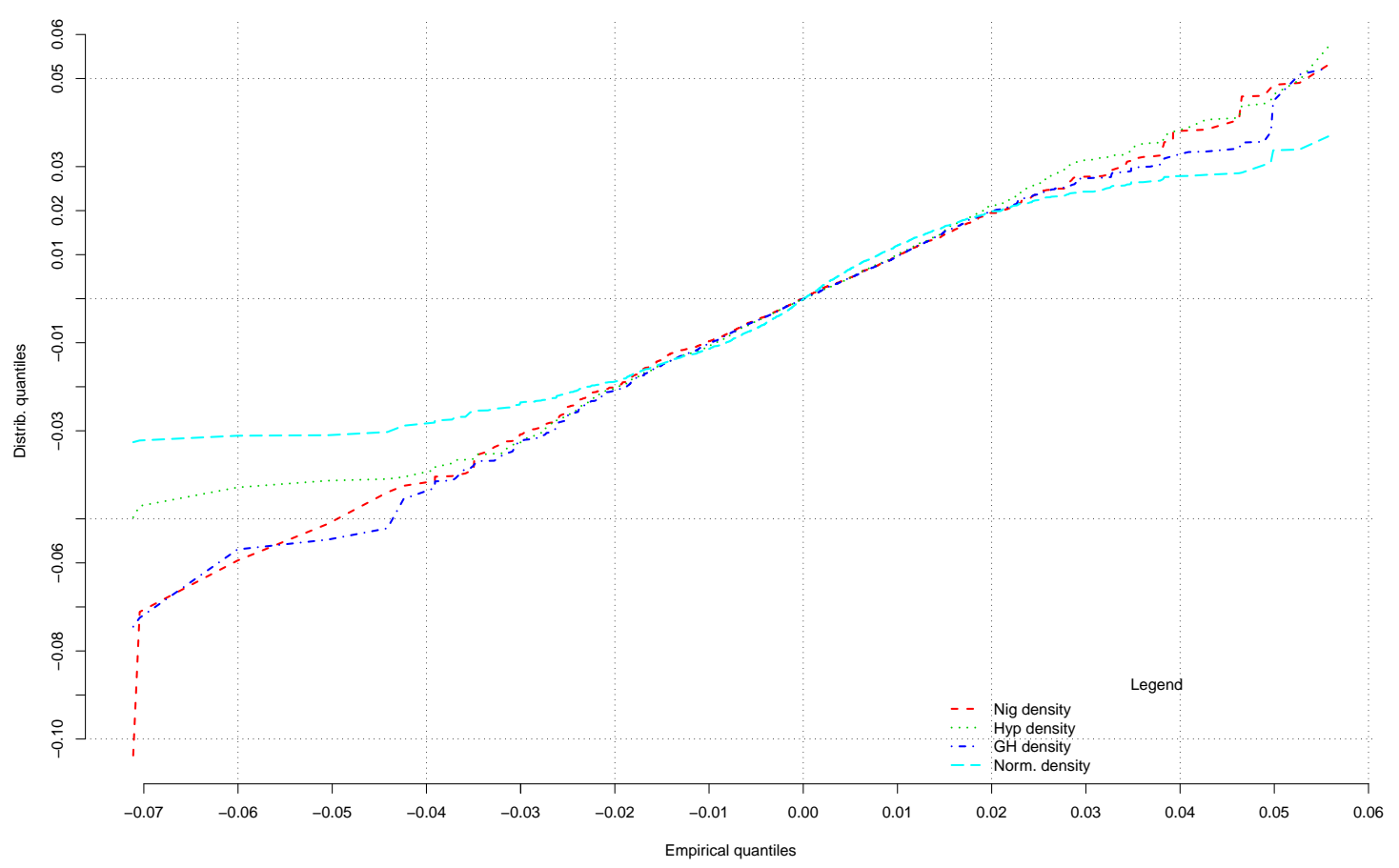

Figure 2: QQ-Plot

This figure presents the qq-plots comparing the empirical quantiles of the CAC 40 and the SP 500 returns vs. the estimated quantiles obtained with the NIG (red), Hyperbolic (green), Generalized Hyperbolic (dark blue) and Normal (light blue) distributions. Time varying variance has been filtered out using an EGARCH process. The samples start on January 2, 1988 and end on October 26, 2007. 